18,12

\title{
Структура и динамика цепочек водородных связей молекул фтористого водорода внутри углеродных нанотрубок
}

\author{
(C) А.В. Савин ${ }^{1,2}$, О.И. Савина ${ }^{2}$ \\ ${ }^{1}$ Федеральный исследовательский центр химической физики им. Н.Н. Семенова РАН, \\ Москва, Россия \\ ${ }^{2}$ Российский экономический университет им. Г.В. Плеханова, \\ Москва, Россия \\ E-mail: asavin@center.chph.ras.ru
}

Поступила в Редакцию 3 апреля 2020 г.

В окончательной редакции 3 апреля 2020 г.

Принята к публикации 25 июня 2020 г.

Методом молекулярной динамики показано, что молекулы фтористого водорода внутри одностенных углеродных нанотрубок с диаметром $D<0.85 \mathrm{~nm}$ образуют плоские зигзагообразные цепочки водородных связей $\mathrm{F}-\mathrm{H} \cdots \mathrm{F}-\mathrm{H} \cdots \mathrm{F}-\mathrm{H} \cdots$. Цепочки, наиболее близкие по структуре к цепочке водородных связей гидроксильных групп ОН, образуют молекулы фтороводорода внутри нанотрубок с индексом хиральности $(6,6)$ и $(10,0)$. В таких открытых нанотрубках с суженными краями цепочки водородных связей $(\mathrm{FH})_{N}$ могут полностью заполнять их внутреннюю полость, образуя структуру, устойчивую к тепловым колебаниям в широком диапазоне температур. В цепочках могут существовать стационарные локализованные на 3-4 звеньях цепи ориентационные дефекты, разделяющие части цепи, имеющие противоположное направление молекул FH. Молекулярные комплексы $(\mathrm{FH})_{N} \in \mathrm{CNT}(6,6)$ и $(\mathrm{FH})_{N} \in \mathrm{CNT}(10,0)$ могут выполнять роль протонопроводящих нанопроводов, в которых внешняя нанотрубка выполняет роль обмотки (изоляции), защищающей и стабилизирующей внутреннюю протонопроводящую цепочку $(\mathrm{FH})_{N}$.

Ключевые слова: нанотрубки, цепочки водородных связей, фтористый водород, транспорт протонов.

DOI: 10.21883/FTT.2020.11.50077.076

\section{1. Введение}

В несколько последних десятилетий молекулярные системы с цепочками водородных связей стали объектом многих исследований в физике, химии и биологии. Основной целью этих исследований является транспорт протонов, происходящий вдоль цепей и решеток (сеток) водородных связей [1-3]. Молекулярные системы с такими цепочками обладают высокой протонной проводимостью [4]. Цепочки водородных связей $\cdots \mathrm{O}-\mathrm{H} \cdots \mathrm{O}-\mathrm{H} \cdots \mathrm{O}-\mathrm{H} \cdots$ выполняют роль протонных „проводов“, обеспечивая эффективный путь для быстрого переноса протонов $[5,6]$.

Экспериментальное и теоретическое изучение углеродных нанотрубок, заполненных водой, показало, что молекулы воды могут заходить внутрь открытых нанотрубок и образовывать там протяженные цепочки водородных связей [7-9]. Механизмы транспорта протонов по таким цепочкам рассмотрены в работах $[6,10]$. УГлеродные нанотрубки гидрофобны, поэтому нахождение внутри них молекул воды энергетически не выгодно. Так внутри нанотрубки с индексом хиральности $(6,6)$ (диаметр $0.80 \mathrm{~nm}$ ) может быть расположена только одна зигзагообразная цепочка молекул воды, в которой каждая молекула участвует в образовании двух водородных связей (в объемной фазе каждая молекула участвует в образовании четырех связей) [11].
Молекулы фтороводорода FH тоже могут образовывать зигзагообразные цепочки водородных связей $(\mathrm{FH})_{\infty}: \cdots \mathrm{F}-\mathrm{H} \cdots \mathrm{F}-\mathrm{H} \cdots \mathrm{F}-\mathrm{H} \cdots . \quad$ При температуpe $T<189.6 \mathrm{~K}$ фтороводород имеет кристаллическую структуру, образованную параллельными плоскими цепочками водородных связей [12]. При высоких температурах длинные цепочки становятся неустойчивыми, но их можно сделать стабильными, если поместить внутрь углеродной нанотрубки (УНТ).

В каждой цепочке $(\mathrm{FH})_{\infty}$ молекула фтороводорода участвует в образовании двух водородных связей. Анализ взаимодействия димера $\mathrm{F}-\mathrm{H} \cdots \mathrm{F}-\mathrm{H}$ с УНТ показывает, что димеру фтороводорода энергетически более выгодно находиться внутри нанотрубки, чем снаружи [13]. Поэтому цепочки $(\mathrm{FH})_{\infty}$ могут быть легко помещены внутрь открытых УНТ и образовывать там устойчивые структуры. Хранение фтороводорода внутри нанотрубок может позволить избежать проблем, связанных с его высокой токсичностью [14].

В настоящей работе будет проведено моделирование динамики конечных цепочек водородных связей $(\mathrm{FH})_{N_{f}}$ $\left(N_{f}\right.$ - число молекул в цепи), помещенных внутрь одностенных углеродных нанотрубок CNT $(n, m)$. Будет показано, что при индексе хиральности нанотрубки $(n, m)=(5,5),(6,6),(9,0),(10,0)$ (при диаметре $D=0.68, \quad 0.80,0.70,0.77 \mathrm{~nm})$ молекулы фтористого водорода образуют внутри нее плоскую зигзагообразую цепочку водородных связей, близкую по своим парамет- 
рам цепочке водородных связей гидроксильных групп $(\mathrm{OH})_{\infty}$. Внутренняя цепочка, устойчивая к тепловым колебаниям в широком диапазоне температур, может быть использована как эффективный путь для переноса протонов. В такой системе $(\mathrm{FH})_{N_{f}} \in \mathrm{CNT}(n, m)$ нанотрубка выполняет роль обмотки (изоляции), защищающей и стабилизирующей протонопроводящий „нанопровод“ $(\mathrm{FH})_{N_{f}}$.

\section{2. Модель}

Деформацию валентной связи двухатомной молекулы FH опишем гармоническим потенциалом

$$
V(\rho)=\frac{1}{2} K\left(\rho-\rho_{0}\right)^{2},
$$

где $\rho$ и $\rho_{0}$ - текущая и равновесная длина связи, $K-$ жесткость связи. Для связи $\mathrm{F}-\mathrm{H}, \rho_{0}=0.929 \AA$, $K=444.3 \mathrm{~N} / \mathrm{m}$.

Для моделирования динамики жидкого фтористого водорода используется [15] потенциал 12-6-1

$$
W_{h b}=\sum_{i_{1}=1}^{3} \sum_{i_{1}=1}^{3} \kappa q_{i_{1}} q_{i_{2}} / r_{i_{1} i_{2}}+4 \epsilon\left[(\sigma / r)^{12}-(\sigma / r)^{6}\right] \text {, }
$$

где первая сумма определяет кулоновское взаимодействие между системами точечных зарядов $\left\{q_{i_{1}}\right\}_{i_{1}=1}^{3}$, $\left\{q_{i_{2}}\right\}_{i_{2}=1}^{3}$, аппроксимирующими распределение зарядов в паре взаимодействующих молекул $\mathrm{FH}\left(r_{i_{1} i_{2}}-\right.$ расстояние между зарядом $q_{i_{1}}$ первой молекулы и зарядом $q_{i_{2}}$ второй молекулы, $\left.i_{1}, i_{2}=1,2,3\right), r$ - расстояние между атомами фтора, коэффициент $\kappa=14.400611 \mathrm{eVA} / e^{2}$. Два положительных заряда $q_{2}, q_{3}$ находятся на атомах $\mathrm{F}$ и Н, отрицательный заряд $q_{1}=-\left(q_{2}+q_{3}\right)$ находится на расстоянии $r_{1}$ от атома $\mathrm{F}$ на отрезке $[\mathrm{FH}]$. Если в потенциале (2) не фиксировать положение двух зарядов на атомах молекулы, то потенциал будет задаваться семью параметрами: двумя зарядами, тремя расстояниями $\left\{r_{i}\right\}_{i=1}^{3}$, задающими положение зарядов на линии, соединяющей атомы $\mathrm{F}$ и $\mathrm{H}$, и двумя параметрами $\epsilon$ и $\sigma$ потенциала Ленарда-Джонса.

Относительная простота и достаточно большое число свободных параметров потенциала 12-6-1 делает его очень удобным для моделирования динамики многомолекулярных комплексов двухатомных молекул FH. Молекула FH обычно участвует в образовании двух сильных водородных связей, значительно при этом поляризуясь. Будем считать, что при изменении длины валентной связи $r=|\mathrm{FH}|$ также пропорционально меняются расстояния расположения зарядов от центра атома фтора. Для моделирования динамики цепочки водородных связей $(\mathrm{FH})_{\infty}$ лучше всего использовать потенциал (2) с параметрами

$$
\begin{gathered}
q_{1}=-0.6397 e, \quad q_{2}=0.6159 e, \quad q_{3}=0.0238 e, \\
r_{1}=0.2577 \rho / \rho_{0}, \quad r_{2}=0.9356 \rho / \rho_{0}, \quad r_{3}=-1.6237 \rho / \rho_{0},
\end{gathered}
$$

$$
\epsilon=0.0079778 \mathrm{eV}, \quad \sigma=2.837109 \AA,
$$

где $\rho$ и $\rho_{0}-$ текущая и равновесная длина валентной связи $\mathrm{F}-\mathrm{H}, e-$ - заряд электрона, размерность $\left[r_{i}\right]=\AA$. При этом наборе параметров молекула FH будет иметь правильные значения дипольного $\mu=q_{1} r_{1}+q_{2} r_{2}+q_{3} r_{3}$ и квадрупольного момента $Q=\sum_{i=1}^{3} q_{i}\left(r_{1}-r_{m}\right)^{2}\left(r_{m}-\right.$ расстояние от центра тяжести молекулы до центра атома $F)$, а энергии и конфигурации стационарных состояний димера $(\mathrm{FH})_{2}$ лучше всего совпадают с экспериментальными данными [16] и результатами квантовых расчетов [17].

Для моделирования динамики УНТ воспользуемся силовым полем, в котором разными потенциалами описываются деформации валентных связей и углов, торсионных и двугранных углов и невалентные взаимодействия пар атомов $[18,19]$. В этой модели энергия деформации валентных связей $\mathrm{C}-\mathrm{C}, \mathrm{C}-\mathrm{H}$ описывается гармоническим потенциалом (1), где для связи $\mathrm{C}-\mathrm{C} \rho_{0}=1.418 \AA, K=508.9 \mathrm{~N} / \mathrm{m}$, для связи $\mathrm{C}-\mathrm{H}$ $\rho_{0}=1.08 \AA, K=444.3 \mathrm{~N} / \mathrm{m}$.

Энергия деформации валентных углов $\mathrm{C}-\mathrm{C}-\mathrm{C}$, $\mathrm{C}-\mathrm{C}-\mathrm{H}$ описывается потенциалом

$$
U\left(u_{1}, u_{2}, u_{3}\right)=U(\varphi)=\epsilon_{a}\left(\cos \varphi-\cos \varphi_{0}\right),
$$

где косинус валентного угла $\cos \varphi=-\left(v_{1} v_{2}\right) /\left|v_{1}\right|\left|v_{2}\right|$, вектора $v_{1}=u_{2}-u_{1}, v_{2}=u_{3}-u_{2}$, а вектора $u_{1}, u_{2}, u_{3}$ задают координаты атомов, образующих валентный угол. Для угла CCC энергия $\epsilon_{a}=1.3143 \mathrm{eV}$, для угла $\mathrm{CCH}-$ $\epsilon_{a}=0.8 \mathrm{eV}$, равновесное значение угла $\varphi_{0}=120^{\circ}$.

Деформации торсионных и двугранных углов описываются потенциалом

$$
W_{t}\left(u_{1}, u_{2}, u_{3}, u_{4}\right)=\epsilon_{t}(1-z \cos \phi)
$$

где $\cos \phi=\left(v_{1}, v_{2}\right) /\left|v_{1}\right|\left|v_{2}\right|$, вектора $v_{1}=\left(u_{2}-u_{1}\right) \times\left(u_{3}-u_{2}\right)$, $v_{2}=\left(u_{3}-u_{2}\right) \times\left(u_{4}-u_{3}\right)$, множитель $z=1$ для двугранного угла (равновесное значение угла $\left.\phi_{0}=0\right)$ и для торсионного угла $\left(\phi_{0}=\pi\right)$, энергия $\epsilon_{t}=0.499 \mathrm{eV}$ (трехмерные вектора $u_{1}, \ldots, u_{4}$ задают координаты атомов, образующих угол). Более детальное описание энергии деформации торсионных и двугранных углов дано в [19].

Невалентное взаимодействие атомов молекулы фтористого водорода с атомами УНТ опишем потенциалом 9-6 из силового поля РСFF

$$
W_{l j}=\epsilon_{l j}\left[2\left(r_{l j} / r\right)^{9}-3\left(r_{l j} / r\right)^{6}\right],
$$

где $r$ - расстояние между взаимодействующими атомами. Для пары атомов F, C энергия взаимодействия $\epsilon_{l j}=0.00217 \mathrm{eV}$, равновесное расстояние $r_{l j}=3.712 \AA$, для пары $\mathrm{H}, \mathrm{C}$ энергия $\epsilon_{l j}=0.00110 \mathrm{eV}$, расстояние $r_{l j}=3.669 \AA$. 


\section{3. Стационарные состояния цепочек (FH) $)_{N_{f}}$ внутри нанотрубок}

Рассмотрим открытые нанотрубки со структурой „кресло“ и „зигзаг“ - см. рис. 1 и 2. К открытым краям (к краевым атомам углерода) присоединим атомы водорода. Для нахождения стационарного состояния системы из цепочки $(\mathrm{FH})_{N_{f}}$ и УНТ нужно решить задачу на минимум потенциальной энергии системы

$$
E \rightarrow \min :\left\{u_{n}\right\}_{n=1}^{N},
$$

где $N$ - общее число атомов С, H, F системы, $u_{n}$ - трехмерный вектор, задающий координаты $n$-го атома, $E$ - общая потенциальная энергия молекулярной системы (сумма всех потенциалов взаимодействия (1), . , (5) для всех атомов системы).

Задача на минимум (6) решалась численно методом сопряженного градиента. Выбирая начальное положение атомов, мы можем получить все основные стационарные состояния системы. При положении цепи $(\mathrm{FH})_{N_{f}}$ вдоль внешней поверхности нанотрубки получается плоская зигзагообразная цепочка водородных связей с углом зигзага $\alpha=117^{\circ}$ и шагом (расстоянием между соседними атомами фтора) $a=|\mathrm{FF}|=2.50 \AA$. Длина валентной связи в цепочке $|\mathrm{FH}|=0.97 \AA$ (для изолированной молекулы $\left.|\mathrm{FH}|=\rho_{0}=0.929 \AA\right)$.

Решение задачи (6) показало, что цепочка водородных связей сохраняет плоскую зигзагообразную форму при ее помещении внутрь нанотрубок диаметра $D<0.85 \mathrm{~nm}-$ см. рис. 1 и 2. С изменением диаметра нанотрубки будет только меняться угол $\alpha$ (угол FFF) и шаг $a$ (расстояние $|\mathrm{FF}|)$ плоского зигзага цепи водородных связей $\mathrm{F}-\mathrm{H} \cdots \mathrm{F}-\mathrm{H} \cdots \mathrm{F}-\mathrm{H} \cdots$. Внутри УНТ $(5,5)$ $(D=0.68 \mathrm{~nm})$ зигзаг цепи будет иметь угол $\alpha=140^{\circ}$, шаг $a=2.49 \AA$. Перемещение цепи с внешней поверхности внутрь для этой нанотрубки приводит к энергетическому выигрышу на $\Delta E=0.073 \mathrm{eV}$ для каждой молекулы цепи. Для цепи внутри УНТ $(6,6)(D=0.80 \mathrm{~nm})$ угол зигзага $\alpha=120^{\circ}$, шаг $a=2.49 \AA$, энергетический выигрыш $\Delta E=0.111 \mathrm{eV}$. Внутри более широкой УНТ $(7,7)$ $(D=0.94 \mathrm{~nm})$ цепь теряет свою плоскую форму и принимает вид плотной трехмерной структуры - см. рис. 1,c. Для цепи внутри УНТ $(9,0)(D=0.70 \mathrm{~nm})$ $\alpha=135^{\circ}, a=2.50 \AA, \Delta E=0.101 \mathrm{eV}$, для цепи внутри УНТ $(10,0)(D=0.77 \mathrm{~nm}) \alpha=122^{\circ}, a=2.49 \AA$, $\Delta E=0.117 \mathrm{eV}$. Внутри более широкой УНТ $(11,0)$ $(D=0.852 \mathrm{~nm})$ цепочка принимает форму трехмерной спирали, идущей вдоль внутренней поверхности нанотрубки - см. рис.21,c. Проведенное моделирование показывает, что цепочку водородных связей, наиболее близкую по структуре к цепочке водородных связей гидроксильных групп $(\mathrm{OH})_{\infty}$, образуют молекулы фтористого водорода внутри нанотрубок с индексом хиральности $(6,6)$ и $(10,0)$, поэтому в структурах $(\mathrm{FH})_{N_{f}} \in \mathrm{CNT}(6,6)$ и $(\mathrm{FH})_{N_{f}} \in \mathrm{CNT}(10,0)$ следует ожидать наиболее высокой протонной проводимости. Рассмотрим более детально эти комплексы.

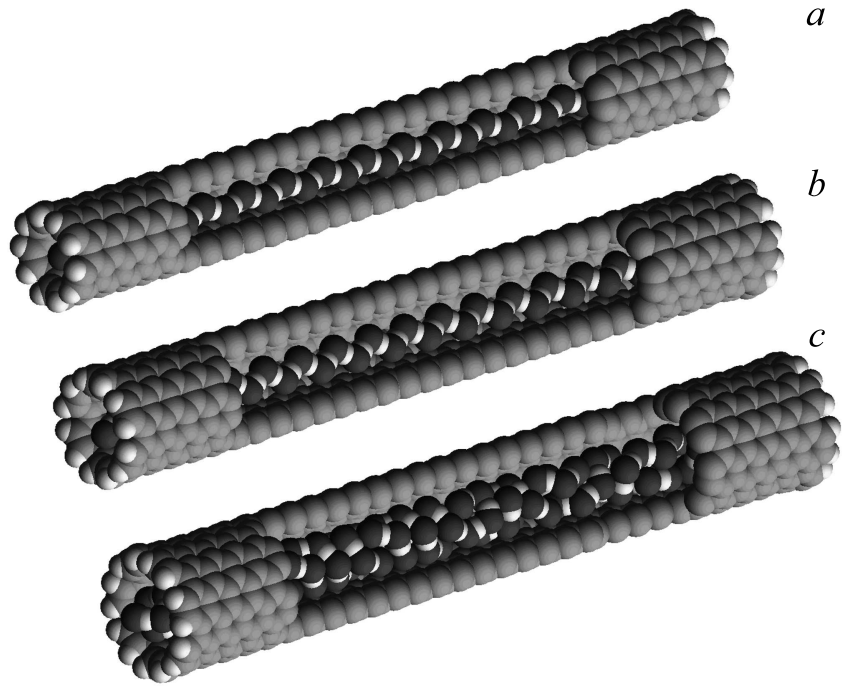

Рис. 1. Стационарное состояние цепочки водородных связей $(\mathrm{FH})_{N_{f}}$ внутри открытой одностенной УНТ со структурой „кресло“: (a) с индексом хиральности $(5,5), N_{f}=32 ;(b)$ с индексом $(6,6), N_{f}=36 ;(c)$ с индексом $(7,7), N_{f}=106$. При длине $L=7.34 \mathrm{~nm}$ нанотрубки имеют химические формулы $\mathrm{C}_{600} \mathrm{H}_{20}, \mathrm{C}_{720} \mathrm{H}_{24}, \mathrm{C}_{840} \mathrm{H}_{28}$. Серые шарики показывают атомы углерода, белые - водорода, черные - фтора. Для удобства вида на цепочку атомов фтористого водорода удалена часть центральных атомов нанотрубки.

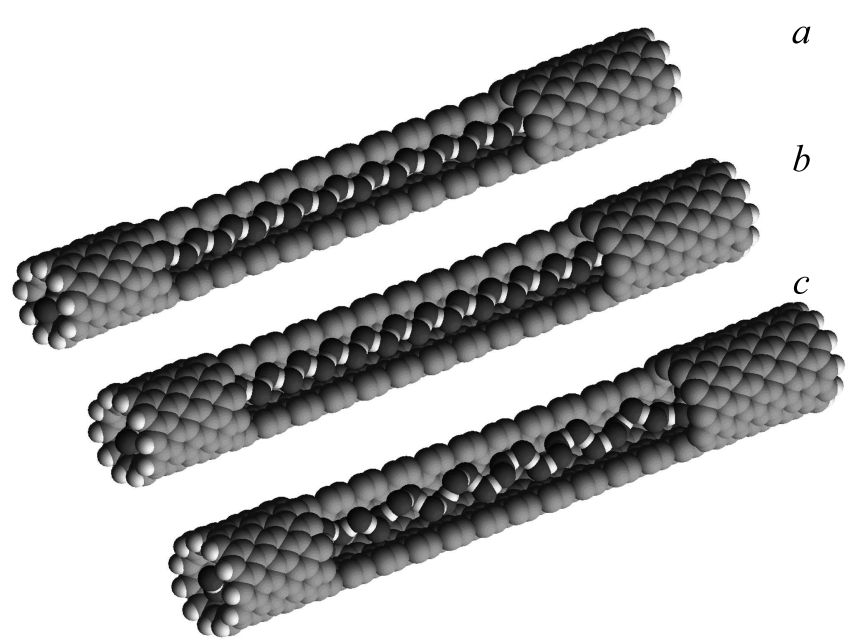

Рис. 2. Стационарное состояние цепочки водородных связей $(\mathrm{FH})_{N_{f}}$ внутри открытой одностенной УНТ со структурой „зигзаг": (a) с индексом хиральности $(9,0), N_{f}=35 ;(b)$ с индексом $(10,0), N_{f}=38 ;(c)$ с индексом $(11,0), N_{f}=44$. При длине $L=7.61 \mathrm{~nm}$ нанотрубки имеют химические формулы $\mathrm{C}_{648} \mathrm{H}_{18}, \mathrm{C}_{720} \mathrm{H}_{20}, \mathrm{C}_{792} \mathrm{H}_{22}$.

Для того, чтобы избежать возможности выхода молекул высокотоксичного фтороводорода из нанотрубок, их открытые края лучше сузить, как показано на рис. 3 и 4. Малый диаметр краевых отверстий $(D=0.47$ и $0.42 \mathrm{~nm})$ не позволит выйти крупному атому фтора, но сохранит возможность выхода и входа протонов. 


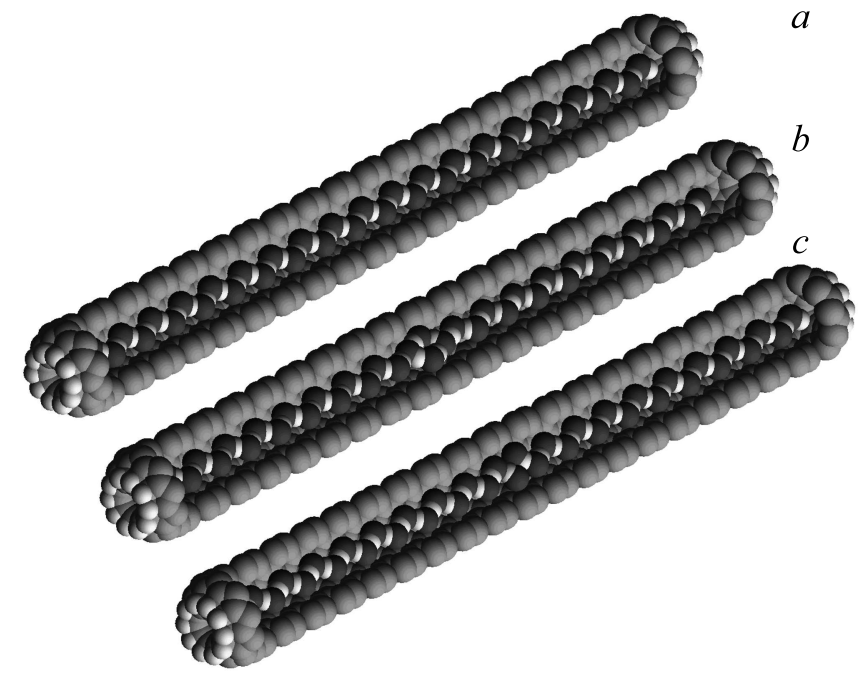

Рис. 3. Стационарные состояния цепочки водородных связей $(\mathrm{FH})_{38}$ внутри одностенной УНТ $(6,6)$ с суженными открытыми краями: (a) основное состояние цепи (все молекулы $\mathrm{FH}$ направлены в одну сторону, слева направо); $(b)$ цепочка с положительным ориентационным дефектом (у первой половины цепи молекулы направлены слева направо, у второй справа налево); (c) цепочка с отрицательным ориентационным дефектом (у первой половины цепи молекулы направлены справа налево, у второй - слева направо). Длина нанотрубки $L=8.7 \mathrm{~nm}$.

Транспорт протонов по зигзагообразной цепи водородных связей проходит с помощью миграции двух типов дефектов: ионных и ориентационных $[1,20]$. В первой стадии переноса протон двигается в виде ионного дефекта. Происходит последовательное перемещение атомов водорода вдоль линий водородных связей от одного атома фтора к соседнему, переводящее цепочку из состояния …FH $\cdots \mathrm{FH} \cdots \mathrm{FH} \cdots$ в состояние $\cdots$ HF $\cdots$ HF $\cdots$ HF $\cdots$. Во второй стадии по цепочке должен пройти ориентационный дефект (последовательный поворот молекул цепи FH на угол зигзага $\alpha$ ) для перевода цепи в исходную ориентацию.

Цепочка водородных связей внутри нанотрубки бистабильна, все молекулы цепи могут быть направлены либо слева направо, либо справа налево. Ориентационный дефект возникает, когда в первой и во второй части цепи ее молекулы направлены в разные стороны. При направлении молекул навстречу друг другу в области локализации дефекта возникает избыточная плотность протонов, поэтому такой дефект называют положительным - см. рис. $3, b$ и 4, $b$. При направлении молекул друг от друга в области локализации дефекта плотность протонов уменьшается, такой дефект называют отрицательным - см. рис. 3, с и 4, $c$.

Вид стационарных ориентационных дефектов цепи водородных связей $(\mathrm{FH})_{N_{f}}$ внутри УНТ $(6,6)$ и $(10,0)$ показан на рис. 3 и 4. Как видно из рисунков, ориентационные дефекты цепи локализуются на 3-4 звеньях цепи. В нанотрубке $(6,6)$ энергия положительного дефекта (разница энергии цепи с дефектом от энергии основного состояния цепи) $\Delta E_{+}=0.56 \mathrm{eV}$, энергия отрицательного дефекта $\Delta E_{-}=0.51 \mathrm{eV}$. В нанотрубке $(10,0)$ энергии ориентационных дефектов цепи $\Delta E_{+}=0.06 \mathrm{eV}$, $\Delta E_{-}=0.91 \mathrm{eV}$.

\section{4. Динамика цепочек водородных связей}

Для проверки устойчивости цепочки водородных связей $(\mathrm{FH})_{N}$, помещенной внутрь одностенной УНТ, было проведено молекулярно-динамическое моделирование цепочки из $N_{f}=177$ молекул $\mathrm{FH}$, помещенных в УНТ с индексом хиральности $(6,6)$ с суженными открытыми краями. При длине $L=38.7 \mathrm{~nm}$ такая нанотрубка состоит из $N_{c}=3620$ атомов углерода и $N_{h}=20$ атомов водорода, присоединенных к краевым атомам нанотрубки. Зигзагообразная цепочка водородных связей $(\mathrm{FH})_{177}$ полностью заполняет внутреннюю полость нанотрубки. Вид такой нанотрубки длины $L=8.7 \mathrm{~nm}$ показан на рис. 3.

Для моделирования тепловых колебаний структуры $(\mathrm{FH})_{N} \in \mathrm{CNT}(6,6)$ нужно численно проинтегрировать систему уравнений Ланжевена

$$
\begin{gathered}
M_{n} \ddot{u}_{n}=-\frac{\partial}{\partial u_{n}} E-\Gamma M_{n} \dot{u}_{n}-\Xi_{n}, \quad n=1, \ldots, N_{c}+N_{h}, \\
M_{n} \ddot{u}_{n}=-\frac{\partial}{\partial u_{n}} E, \quad n=N_{c}+N_{h}+1, \ldots, N, \quad(7)
\end{gathered}
$$

где $N=N_{c}+N_{h}+2 N_{f}$ - общее число атомов молекулярной структуры, $M_{n}$ - масса $n$-го атома, $u_{n}-$ трехмерный вектор, задающий координаты $n$-го атома, $\Gamma=1 / t_{r}-$ коэффициент трения (время релаксации термостат $\left.t_{r}=1 \mathrm{ps}\right), \Xi_{n}=\left\{\xi_{n, i}\right\}_{i=1}^{3}-$ вектор нормально распределенных случайных сил Ланжевена с функциями корреляции:

$$
\left\langle\xi_{n, i}\left(t_{1}\right) \xi_{k, j}\left(t_{2}\right)\right\rangle=2 M_{n} k_{B} T \Gamma \delta_{n k} \delta_{i j} \delta\left(t_{1}-t_{2}\right)
$$

$\left(k_{B}\right.$ - постоянная Больцмана, $T-$ температура термостата).

При использовании системы уравнений (7) с термостатом Ланжевена будут взаимодействовать только атомы нанотрубки, а внутренняя цепочка $(\mathrm{FH})_{N_{f}}$ будет термализоваться за счет взаимодействия с атомами нанотрубки.

В качестве начального решения системы уравнений движения (7) возьмем стационарное состояние структуры $(\mathrm{FH})_{N_{f}} \in \mathrm{CNT}(6,6)$, полученное при решении задачи на минимум (6). Пусть в начальный момент нанотрубка направлена вдоль оси $x$. Для того, чтобы сохранить направление нанотрубки, зафиксируем положение двух ее атомов: одного атома из ее левого, другого - из ее правого края. Тогда направление $n$-ой молекулы FH будет задаваться углом $\phi_{n}$ между вектором $\mathbf{F H}_{n}$ и осью $x$. В основном состоянии цепи $(\mathrm{FH})_{N_{f}}$, когда все 


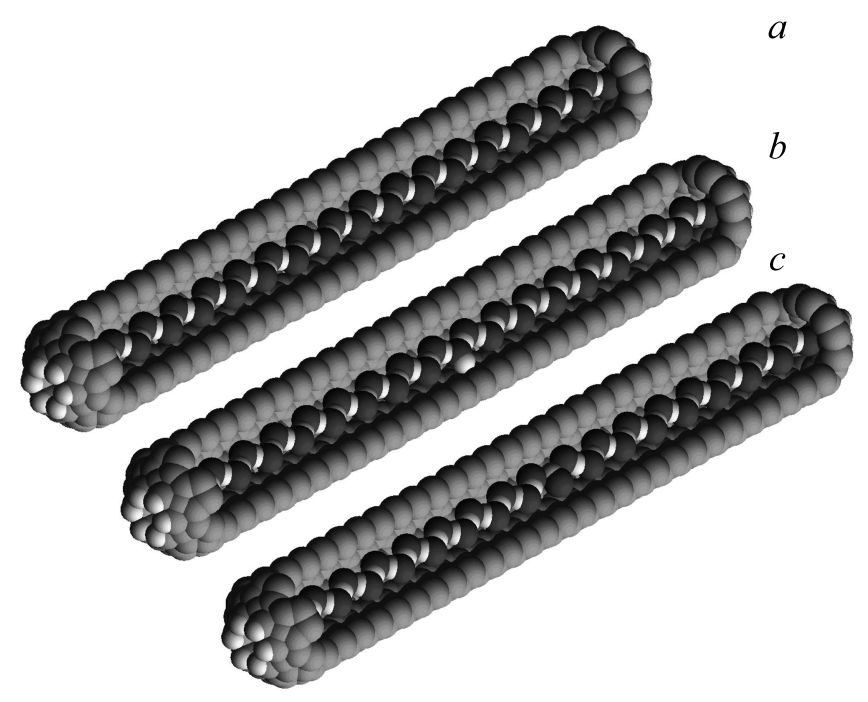

Рис. 4. Стационарные состояния цепочки водородных связей $(\mathrm{FH})_{36}$ внутри одностенной УНТ $(10,0)$ с суженными краями: (a) основное состояние цепи; $(b)$ цепочка с положительным ориентационным дефектом; $(c)$ цепочка с отрицательным ориентационным дефектом. Длина нанотрубки $L=7.47 \mathrm{~nm}$.
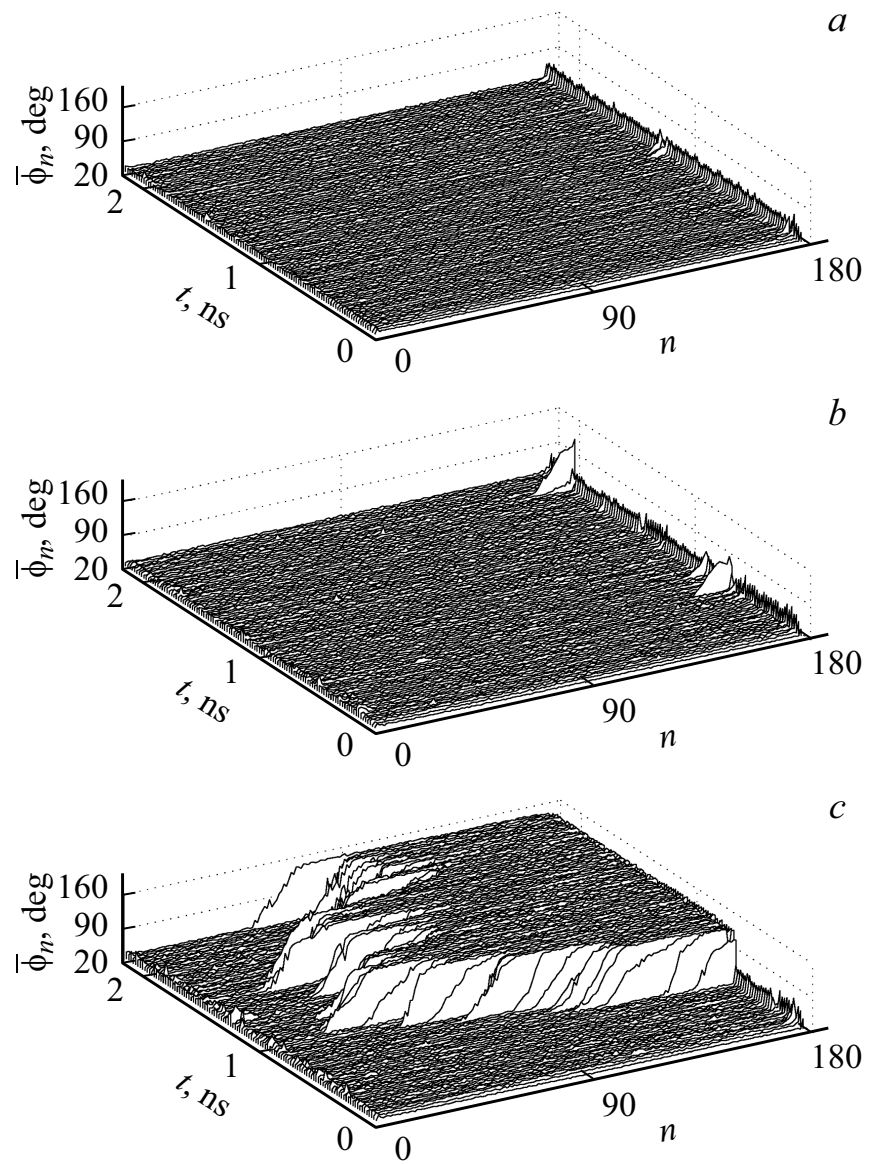

Рис. 5. Зависимость от времени $t$ ориентационных углов молекул FН $\left\{\bar{\phi}_{n}\right\}_{n=1}^{N_{f}}$ для цепи из $N_{f}=177$ молекул, находящихся в УНТ $(6,6)$ с суженными открытыми краями (длина нанотрубки $L=8.7 \mathrm{~nm}$ ). Показана динамика при температуре (a) $T=350 \mathrm{~K},(b) T=400 \mathrm{~K},(c) T=440 \mathrm{~K}$. В начальный момент времени $t=0$ цепочка находилась в основном состоянии $\phi_{n}=\phi_{0}$.
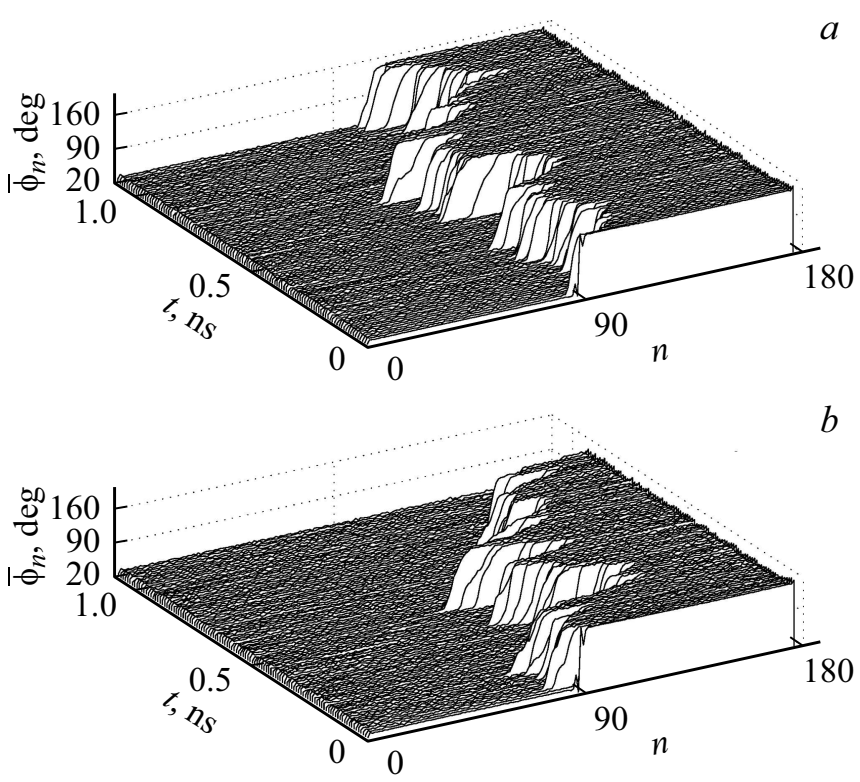

c
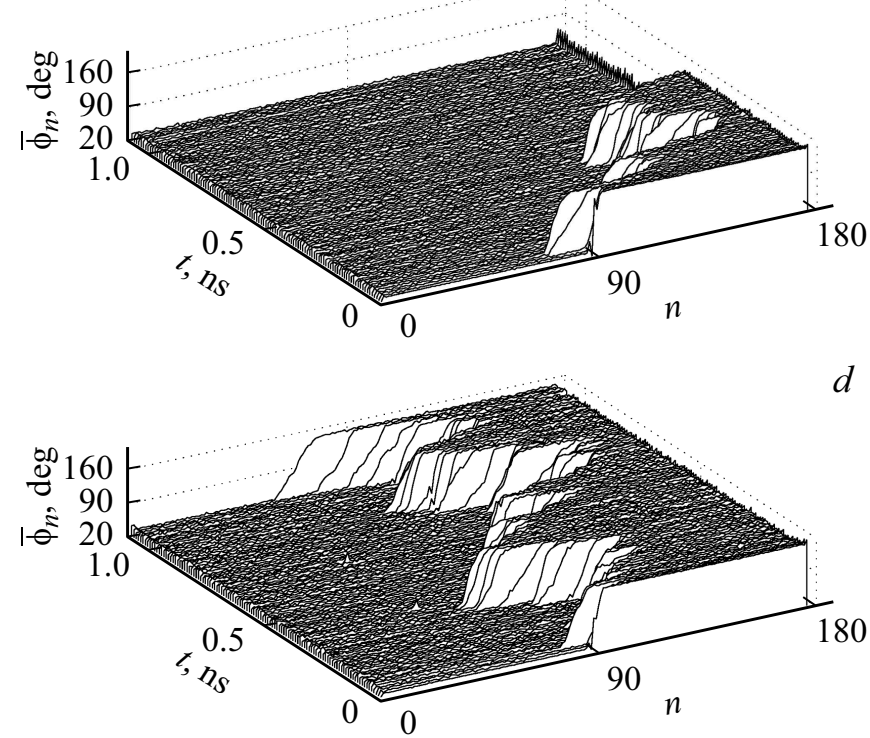

Рис. 6. Динамика в термализованной цепи (FH) 177 положительного ориентационного дефекта. Показана зависимость от времени $t$ ориентационных углов молекул $\left\{\bar{\phi}_{n}\right\}_{n=1}^{177}$ при температуре (a) $T=200 \mathrm{~K},(b) T=250 \mathrm{~K}$, (c) $T=300 \mathrm{~K}$, (d) $T=350 \mathrm{~K}$. В начальный момент времени $t=0$ стационарный дефект находился в центре цепи.

молекулы направлены слева направо, углы $\left\{\phi_{n}=\phi_{0}\right\}_{n=1}^{N_{f}}$ (равновесное значение угла $\phi_{0}=30^{\circ}$ ), а когда все молекулы направлены справа налево, углы $\left\{\phi_{n}=\pi-\phi_{0}\right\}_{n=1}^{N_{f}}$. При наличии в цепи положительного ориентационного дефекта последовательность $\left\{\phi_{n}\right\}_{n=1}^{N_{f}}$ будет иметь вид кинка (в области локализации дефекта углы $\phi_{n}$ будут возрастать от значения $\phi_{0}$ до значения $\pi-\phi_{0}$, рис. 6). При наличии отрицательного ориентационного дефекта последовательность $\left\{\phi_{n}\right\}_{n=1}^{N_{f}}$ будет иметь вид антикинка (в области локализации дефекта углы $\phi_{n}$ будут убывать от значения $\pi-\phi_{0}$ до значения $\phi_{0}$, рис. 7). 
Для того, чтобы сгладить быстрые флуктуации значений углов ориентации $\phi_{n}(t)$, мы будем следить за изменением их усредненных по времени значений

$$
\bar{\phi}_{n}(t)=\frac{1}{t_{1}} \int_{0}^{t_{1}} \phi_{n}(t+\tau) d \tau
$$

с временем усреднения $t_{1}=10 \mathrm{ps}$.

Моделирование динамики

комплекса $(\mathrm{FH})_{177} \in \mathrm{CNT}(6,6)$ показало, что цепочка водородных связей внутри нанотрубки при всех рассмотренных температурах $T<500 \mathrm{~K}$ остается устойчивой - она всегда остается внутри и сохраняет зигзагообразную форму. Динамика ориентационных углов молекул
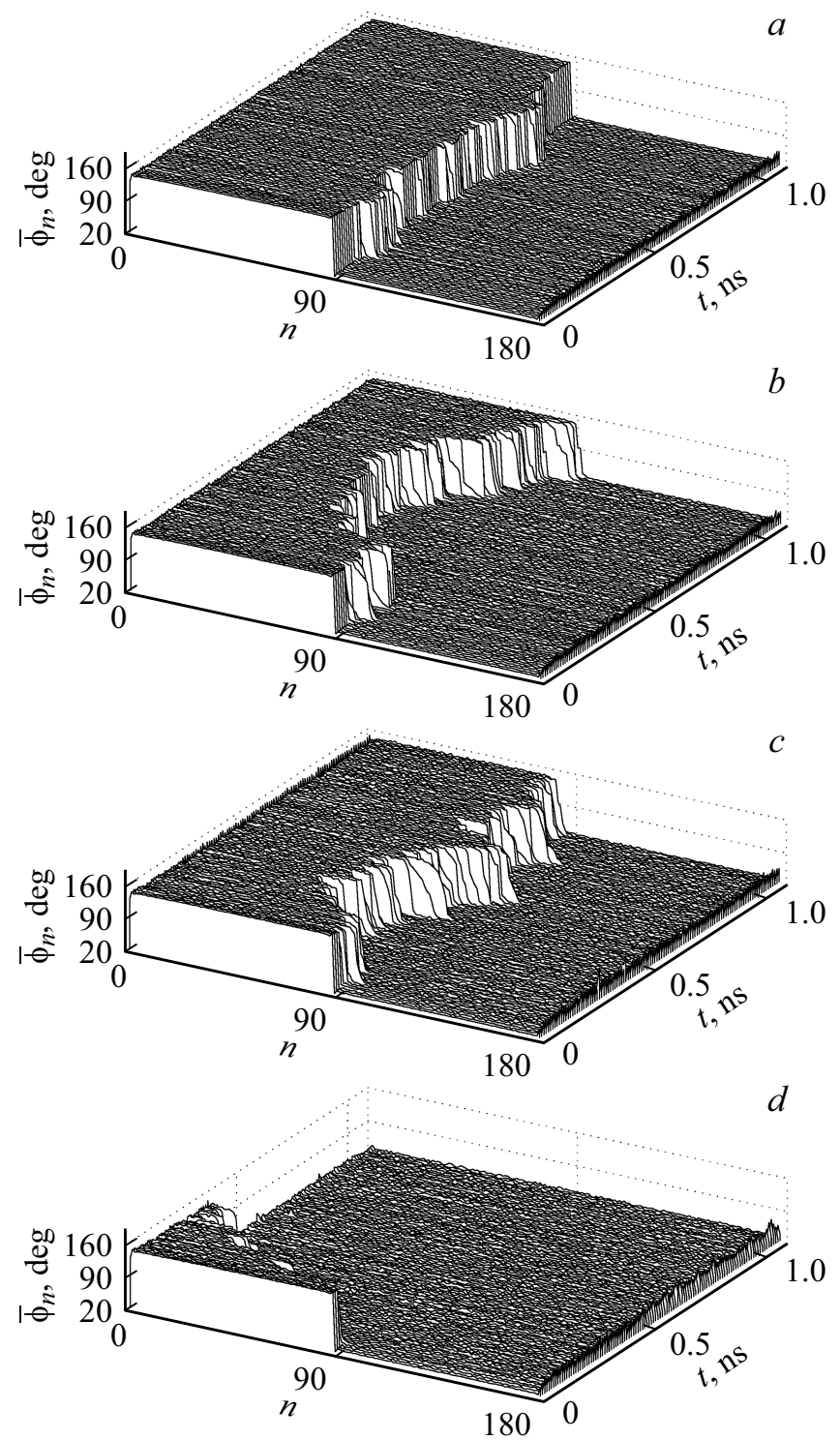

Рис. 7. Динамика в термализованной цепи (FH) 177 отрицательного ориентационного дефекта. Показана зависимость от времени $t$ ориентационных углов молекул $\left\{\bar{\phi}_{n}\right\}_{n=1}^{N_{f}}$ при температуре (a) $T=200 \mathrm{~K},(b) T=250 \mathrm{~K}$, (c) $T=300 \mathrm{~K}$, (d) $T=350 \mathrm{~K}$. В начальный момент времени $t=0$ стационарный дефект находился в центре цепи. цепи $\left\{\bar{\phi}_{n}(t)\right\}_{n=1}^{177}$ при разных значениях температуры представлена на рис. 5. При $T<400 \mathrm{~K}$ цепочка водородных связей всегда остается в начальном основном состоянии $\bar{\phi}_{n} \approx \phi_{0}$. При $T>400 \mathrm{~K}$ на правом конце цепи уже может появляться, а затем двигается по ней положительный ориентационный дефект (кинк).

Моделирование динамики комплекса с ориентационным дефектом внутренней цепи водородных связей показало, что движение дефектов является термически активированным (при низких температурах дефекты остаются пинингованными) и происходит в виде броуновского движения частицы вдоль цепи. Чем выше температура, тем выше подвижность дефектов - см. рис. 6 и 7. Положительный ориентационный дефект (кинк) обладает более высокой подвижностью, чем отрицательный дефект (антикинк). При выходе дефекта на конец цепи он исчезает, и цепочка переходит в однородное основное состояние.

Таким образом, тепловые колебания не приводят к разрывам цепочки водородных связей $(\mathrm{FH})_{\infty}$ внутри углеродной нанотрубки, поэтому цепочка всегда может быть использована для транспорта протонов. При переносе протона по цепи водородных связей самое большое время требуется на ее переориентацию после прохождения ионного дефекта. Такая переориентация, переход цепи из состояния НF $\cdots$ HF $\cdots$ HF $\cdots$ в состояние $\mathrm{FH} \cdots \mathrm{FH} \cdots \mathrm{FH} \cdots$, происходит за счет термически активированного прохождения вдоль цепи положительного ориентационного дефекта. Поэтому тепловые колебания не только не препятствуют транспорту протонов по цепи водородных связей внутри нанотрубки, но являются его необходимым условием.

\section{5. Заключение}

Проведенное численное моделирование показало, что молекулы фтористого водорода внутри одностенных углеродных нанотрубок с диаметром $D<0.85 \mathrm{~nm}$ образуют плоские зигзагообразные цепочки водородных связей $\mathrm{F}-\mathrm{H} \cdots \mathrm{F}-\mathrm{H} \cdots \mathrm{F}-\mathrm{H} \cdots$. Цепочку, наиболее близкую по структуре к цепочке водородных связей гидроксильных групп ОН, образуют молекулы фтороводорода внутри нанотрубок с индексом хиральности $(6,6)$ и $(10,0)$. В таких открытых нанотрубках с суженными краями цепочки водородных связей $(\mathrm{FH})_{N}$ могут полностью заполнять их внутреннюю полость, образуя структуру, устойчивую к тепловым колебаниям в широком диапазоне температур. В цепочках могут существовать стационарные, локализованные на 3-4 звеньях цепи, ориентационные дефекты, разделяющие части цепи, имеющие противоположное направление молекул FH.

Моделирование динамики комплекса $(\mathrm{FH})_{N_{f}} \in \mathrm{CNT}(6,6)$ показало, что тепловые колебания не приводят к разрывам внутренней цепочки водородных связей. Наличие тепловых колебаний является необходимым условием для возможности движения вдоль цепочки локализованных ориентационных дефектов. За 
счет движения дефектов происходит переориентация цепочек водородных связей, необходимая для возможности возобновляемого протонного транспорта. Таким образом, проведенное моделирование показало, что молекулярные комплексы $(\mathrm{FH})_{N_{f}} \in \mathrm{CNT}(6,6)$ и $(\mathrm{FH})_{N_{f}} \in \mathrm{CNT}(10,0)$ могутвыполнять роль протонопроводящих нанопроводов, в которых внешняя нанотрубка выполняет роль обмотки (изоляции), защищающей и стабилизирующей внутреннюю протонопроводящую цепочку $(\mathrm{FH})_{N_{f}}$.

\section{Финансирование работы}

Научно-исследовательская работа выполнена за счет субсидии, выделенной ФИЦ ХФ РАН на выполнение государственного задания, тема 0082-2014-0013. Вычислительные ресурсы предоставлены межведомственным суперкомпьютерным центром РАН.

\section{Конфликт интересов}

Авторы заявляют, что у них нет конфликта интересов.

\section{Список литературы}

[1] J.F. Nagle, S. Tristram-Nagle. J. Membrane Biol. 74, 1-14 (1983).

[2] K.-D. Kreuer. Chem. Mater. 8, 3, 610 (1996).

[3] C. Nagamani, U. Viswanathan, C. Versek, M.T. Tuominen, S.M. Auerbach, S. Thayumanavan. Chem. Commun. 47, 6638 (2011).

[4] G. Zundel. Adv. Chem. Phys. 111, 1 (2000).

[5] F. Fillaux. J. Mol. Struct. 615, 45 (2002).

[6] V.M. Karpan, Y. Zolotaryuk, P.L. Christiansen, A.V. Zolotaryuk. Phys. Rev. E 70, 056602 (2004).

[7] G. Hummer, J. Rasaiah, J. Noworyta. Nature 414, 188 (2001).

[8] C. Dellago, M.M. Naor, G. Hummer. Phys. Rev. Lett. 90, 10, 105902 (2003)

[9] B.H.S. Mendonca, D.N. de Freitas, M.H. Kohler, R.J.C. Batista, M.C. Barbosa, A.B. de Oliveira. Physica A 517, 491 (2019).

[10] J. Chen, X.-Z. Li, Q. Zhang, A. Michaelides, E. Wang. Phys. Chem. Chem. Phys. 15, 6344 (2013).

[11] I. Hanasaki, A. Nakamura, T. Yonebayashi, S. Kawano. J. Phys.: Condens. Matter 20, 015213 (2008).

[12] M. Atoji, W.N. Lipscomb. Acta Crystallographica 7, 173 (1954).

[13] A. Roztoczyńska, J. Koztowska, P. Lipkowski, W. Bartkowiak. Phys. Chem. Chem. Phys. 18, 2417 (2016).

[14] W.F. Gtari, B. Tangour. Acta Chim. Slov. 65, 289 (2018).

[15] M.E. Cournoyer, W.L. Jorgensen. Mol. Phys. 51, 119 (1984).

[16] T.R. Dyke, B.J. Howard, W. Klemperer. J. Chem. Phys. 56, 2442 (1972).

[17] А.В. Немухин. Неэмпирическое изучение динамики молекулярных систем. Журн. физ. химии 66, 4 (1992).

[18] W.D. Cornell, Wendy P. Cieplak, C.I. Bayly, I.R. Gould, K.M. Merz, D.M. Ferguson, D.C. Spellmeyer, T. Fox, J.W. Caldwell, P.A. Kollman. J. Am. Chem. Soc. 117, 19, 5179 (1995).

[19] A.V. Savin, Y.S. Kivshar, B. Hu. Phys. Rev. B 82, 195422 (2010).

[20] L. Onsager. Science 166, 3911, 1359 (1969).

Редактор Ю.Э. Китаев 